\title{
Artificial intelligence and cyber-physical mechanical processing systems in digital industry
}

\author{
Yuri Kabaldin ${ }^{1, *}$, and Dmitrii Shatagin ${ }^{1}$ \\ ${ }^{1}$ Nizhny Novgorod State Technical University n.a. R.E. Alekseev, 603950 Nizhny Novgorod, Russia
}

\begin{abstract}
The article suggests new approaches to the creation and control of cyber-physical machining systems in the digital industry. Features of the use of artificial intelligence and the possibilities of big data for analyzing the current and future state of $\mathrm{CNC}$ machines are considered. A specialized neuromorphic chip is proposed, with the possibilities for deep learning, for control and sharing knowledge between CNC machines as part of a technology group. The features of the use of blockchain technology in digital industry with the use of the industrial Internet of things are shown.
\end{abstract}

\section{Introduction}

Currently, the transition of industrial enterprises to the "digital" production, implemented under the concept of Industry 4.0, One of the main tasks at the same time is the digitization of existing production systems and the creation of fundamentally new technological systems based on CNC machines that can perform high-performance machining at the expense of giving them new distinctive features $[1,2]$, in particular hybrid technologies. The main feature of such systems is the deep integration of physical and computing processes into a single information space that allows to significantly improve the quality, productivity and efficiency of the production system as a whole, by constantly monitoring the technical condition of the equipment and determining its optimal operating modes, ensuring high stability of the cutting processes . Such technological systems should be characterized as cyber-physical systems (CFS).

To realize these possibilities, $\mathrm{CNC}$ machines must be equipped with special sensors and devices capable of collecting, analyzing and exchanging diagnostic information with each other. As studies show, every second technological equipment generates millions of bytes of useful information characterizing its current and future state. However, modern monitoring systems and $\mathrm{CNC}$ racks collect and analyze less than $1 \%$ of the percentage of useful data and this information is not sufficient for an objective analysis of the various processes occurring in the machine. The most informative source of diagnostic data is vibro-acoustic emission, which always accompanies the processes taking place in the machines. However, there are a number of problems when working with this type of

*Corresponding author: uru.40@mail.ru 
signals, characterized by a complex composition and a high degree of noisiness.

Modern approaches in the field of processing large volumes of heterogeneous diagnostic data and the field of automatic control of technical objects are the approaches of artificial intelligence [1-5], in particular artificial neural networks of deep learning realized both at the software and hardware level (neuromorphic chips). The ANN capabilities allow to detect hidden dependencies in large data sets, to recognize and classify digital signals in real time, to accumulate and systematize new knowledge about the object under study, to predict the dynamics of the state of the object, and to determine the optimal regimes.

AI approaches require the constant collection and accumulation of knowledge about ongoing processes, as well as periodic exchange of knowledge between the intellectual units of the production system, which allows to significantly accelerate the learning process of a group of cyber-physical systems.

An indispensable condition for the integration of CFS into production is the creation of an industrial Internet network and distributed databases, for the exchange of information and knowledge between production units through encrypted channels. The modern approach in the field of cryptography and data exchange is the "Block chain" technology [1].

\section{Software implementation}

To analyze the current technical condition of the equipment and optimize the processing modes, it is necessary to obtain a mathematical model of the machine. However, taking into account the multifactorial of the cutting process itself, the heterogeneity of the parameters of the elastic system of the machine, depending on the relative location of the assemblies and the shape of the workpiece to be machined, analytical models are very difficult to obtain, and in production conditions it is completely impossible. It is necessary to take into account the various unique factors and the current wear of the equipment. To solve this problem, it is proposed to use neural network equipment models. Creation of such models is carried out during the training of artificial neural networks (ANN) on real data obtained during the operation of equipment. This takes into account many different factors, such as the shape and material of the workpiece, the geometry of the tool, the position of the tool forming surfaces, etc. The training sample for ANN is presented as two n-dimensional vectors. The first vector is the input vector, characterizes the processing conditions, and the output vector characterizes the corresponding states of the technological system. In this case, the state is recorded at various times and throughout the processing.

As a result, the neural network model [1-6] will allow to take into account a unique dynamic portrait that characterizes the machine's capabilities, processing quality, economy, productivity, current and future technical condition of the equipment. To register signals it is necessary to place sensors at different points of the machine (Fig. 1). Placements are selected based on the analysis of the layout and design of the equipment. As a rule, this is the drive of the main movement and the movement of the feeds, as well as the cutting zone itself. Information on the position of the operating elements, cutting speed and feed rate can be registered directly from the $\mathrm{CNC}$ rack. 


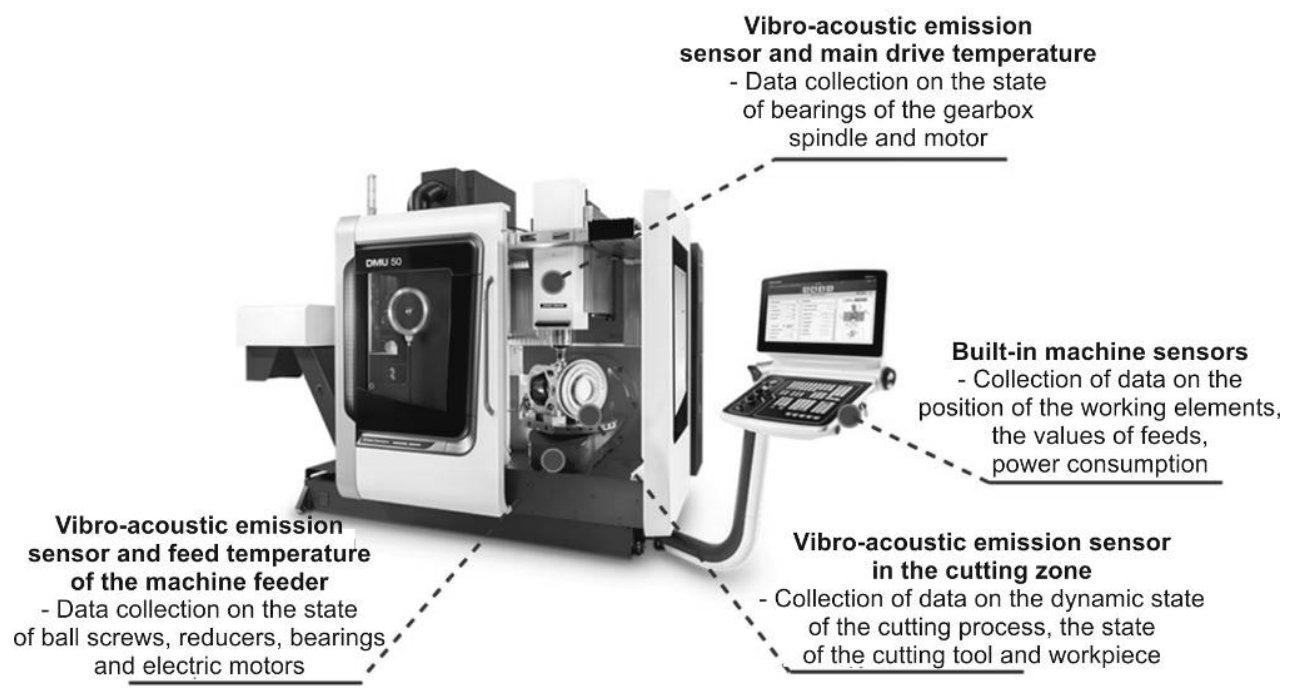

Fig. 1. Placement of sensors on a CNC machine.

The process of obtaining data for learning neural network models should be accompanied by an automatic analysis of redundancy and data insufficiency. For this purpose, a special intellectual dialogue module is provided in the system, which, based on the cluster analysis of the collected data, identifies the "empty" zones of the vector space in the training sample. Also this module can give recommendations for conducting a series of additional qualifying tests to fill such zones or to search in third-party databases (DB). An additional important feature of the system is an assessment of the adequacy of the simulation. After learning the INS, the neural network model (Fig. 2) is able to predict the various conditions of the machine in time.

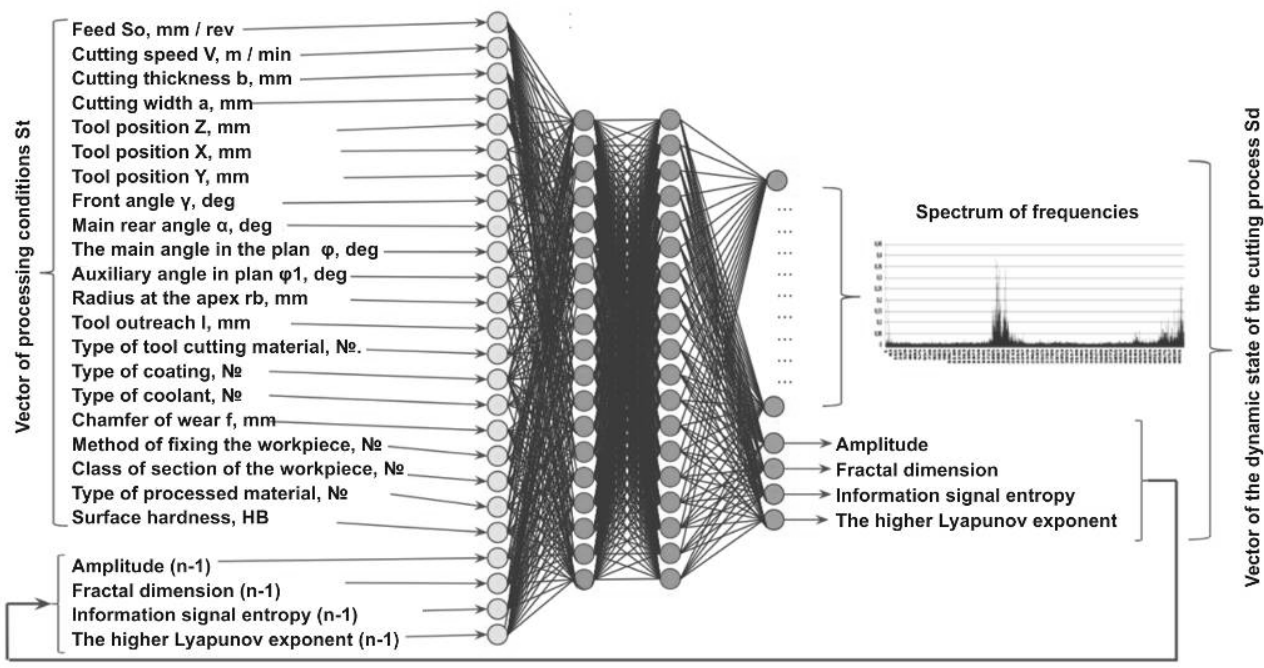

Fig. 2. An example of a neural network model of the dynamic stability of the cutting process.

As a result, at the stage of technological preparation of production the technologist gets access to the neural network model of the machine and downloads the control program from the CAM system for further analysis and optimization. In the automatic mode, the 
shape of the workpiece is analyzed in the working space of the machine on the basis of the developed neural network algorithms for the classification of the point clouds of the $3 \mathrm{D}$ workpiece model (Fig. 3). Then, the obtained data on the shape of the blank and information on the technological conditions are fed to the input of the neural network model of the machine for performing simulation (Fig. 4). Depending on the set step, the system simulates the dynamic state at various points in the tool path, while determining the surface roughness, tool resistance, vibration amplitude and stability. In accordance with the assigned restrictions and target functions, optimization of the modes and processing conditions at each simulated point occurs. At the end of the simulation, an adjusted UE is issued to be sent to the machine in order to identify unfavorable locations and take measures to prevent the marriage.

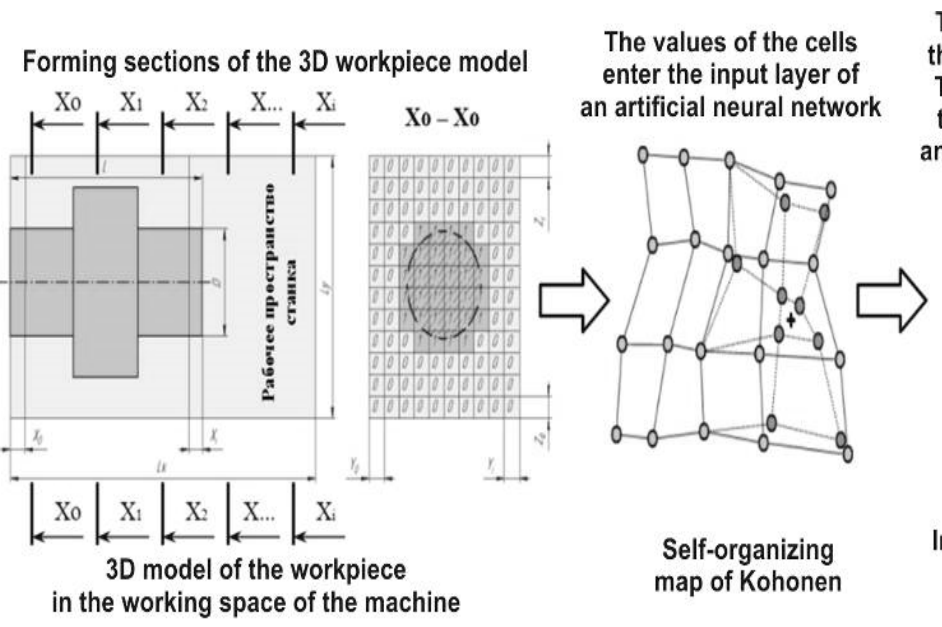

The closest sections form the corresponding classes. There is a formalization of the form of the workpiece and the compression of data

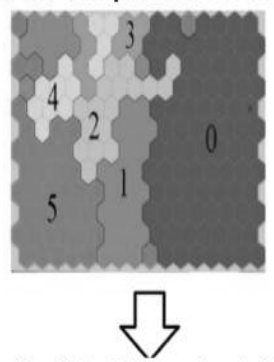

In the DB of dynamic stability of the cutting process

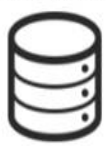

Fig. 3. Intellectual analysis of the form of the workpiece.

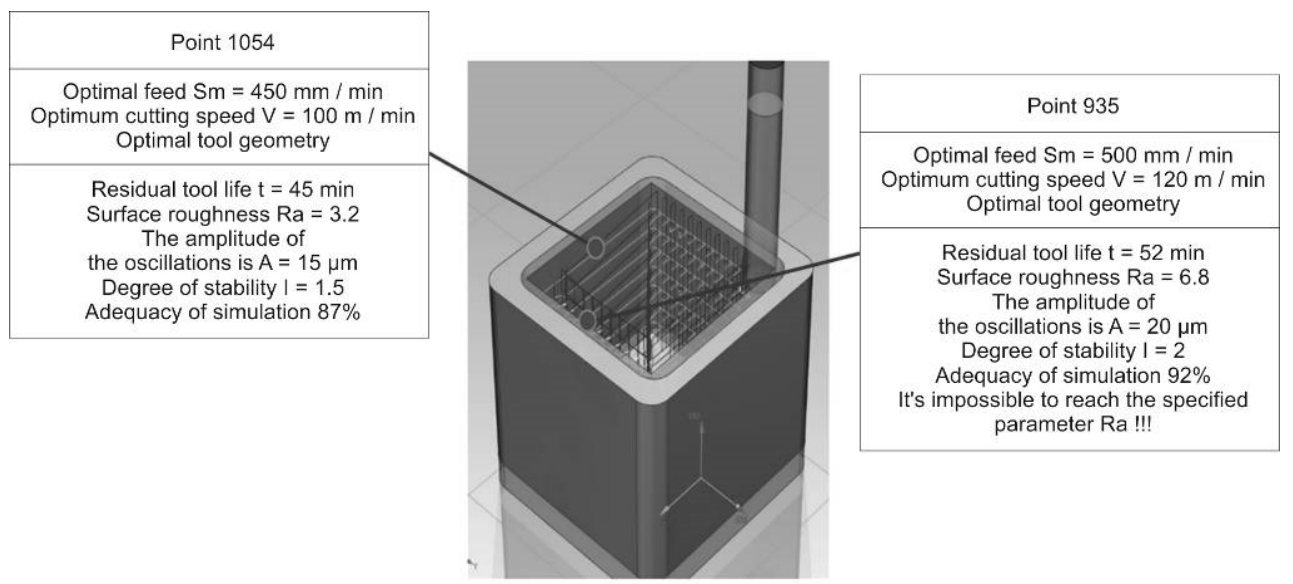

Fig. 4. Simulation modeling of the milling process and optimization of machining modes at various points of the tool trajectory. 


\section{Data storage and processing}

Considering the constant flow of a large array of data, the system uses distributed and cloud storage of the database, while cryptographic protection of all data is carried out. The collected information in the database (Fig. 5) is subjected to a complex analysis using the BigData and DataMining approaches. The regression and cluster analysis of data, using ANN, allows us to identify hidden dependencies and new classes in information arrays. To accelerate the processing processes, the parallel computing capabilities of nVidia CUDA [1-6] are used. Later, using the dialogue module, new identified classes of data and dependencies are refined together with the expert. Thus, the structure of the database implies the creation of digital duplicates of equipment and the enterprise as a whole. This approach allows for large-scale modeling and optimization of various processes taking into account the features of production and equipment.

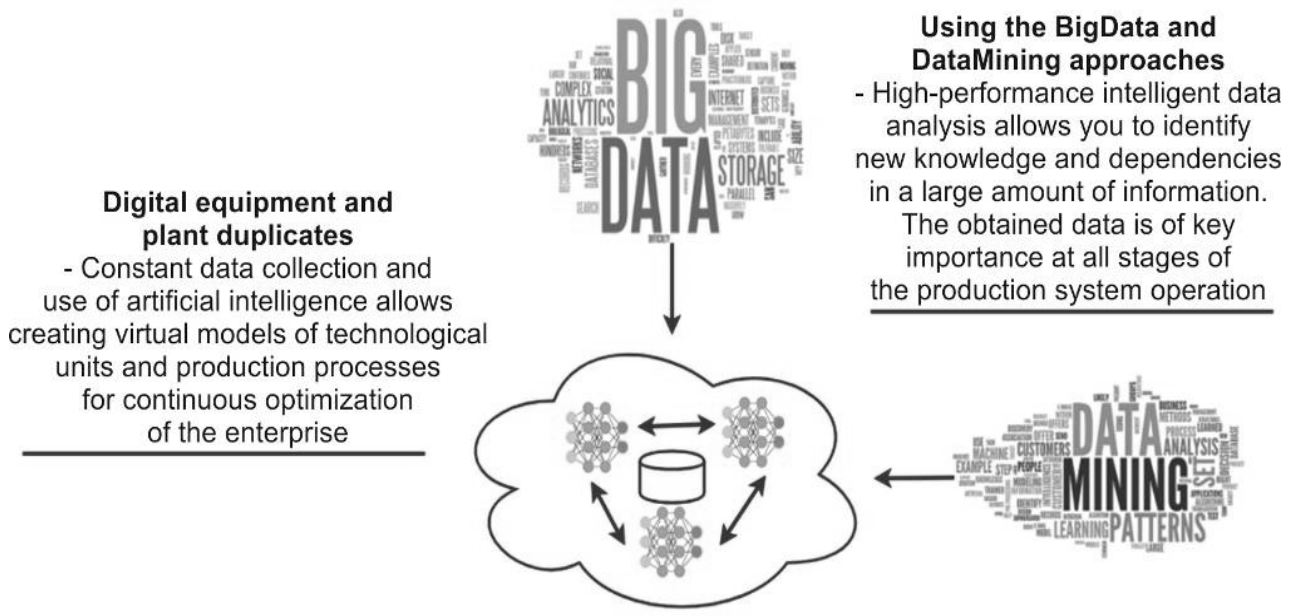

Fig. 5. DB of dynamic stability.

\section{Multi-agent training}

Taking into account the high demand for information on the state of technological processes and equipment, methods of knowledge sharing between the equipment are used for the optimal functioning of this system. The possibility of knowledge sharing is based on an assessment of the degree of similarity of technological units. To do this, the responses of different machines to the same dynamic impact in the course of simulation are compared. If the difference between the responses is within the specified limits, then the systems are recognized as similar and can exchange knowledge (Fig. 6).

The combination of machines by the industrial Internet network [1,7] will allow the targeted search of necessary knowledge for specific processing conditions. All data exchange is carried out on the basis of "Block chain" technology and allows you to track the history of all transactions and prevent unauthorized access to data. This approach allows us to organize fundamentally new production units for the extraction and storage of new knowledge and information about technological processes. The knowledge gained during the work of the CFS has its own value and can be used as a passive source of income. 

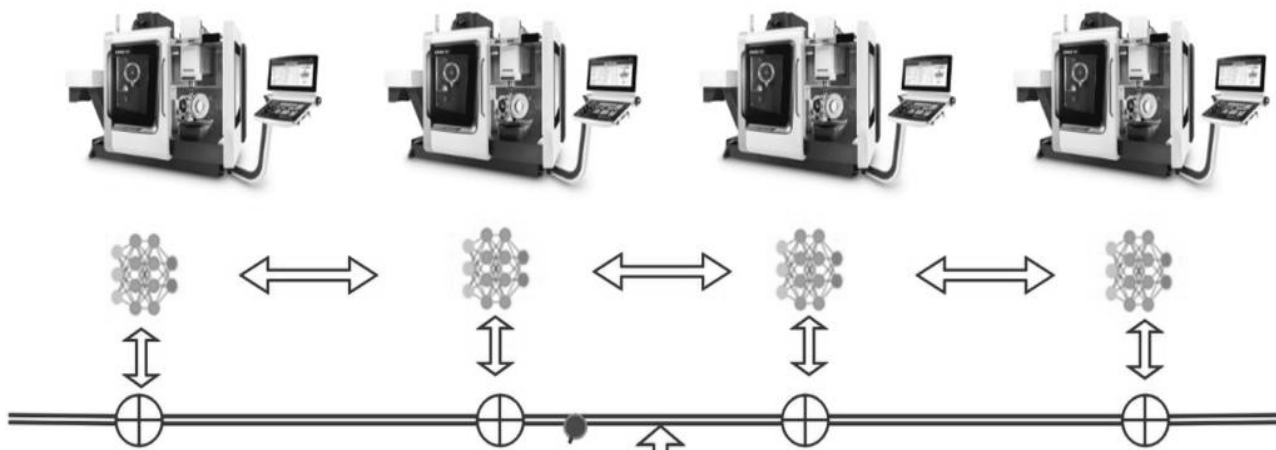

\section{(multi-agent training)}

Sharing knowledge between equipment - Allows to significantly reduce the time of technological preparation of production and accelerate the process of "Data mining"
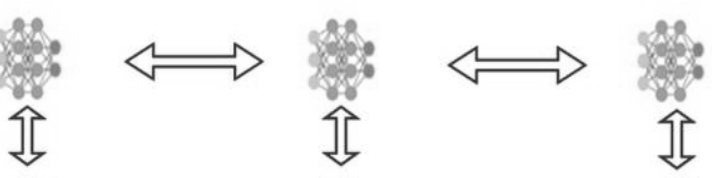

15

I5

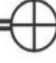

Multi-agent training on a global scale

- The use of the global network and technology "Blockchain"

allows you to share knowledge within international corporations and around the world

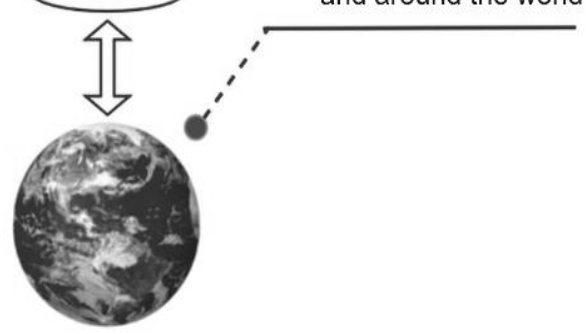

Fig. 6. Sharing knowledge between machine tools

\section{Hardware implementation. Neuromorphic chips}

For the hardware implementation of the indicated methods and approaches, standard communication protocols, wired and wireless networks, industrial data storage servers, as well as special intelligent devices for data collection and processing, are used. These are the neural-morphic controllers (NMCs) developed at the NNSTU R.E. Alekseeva.

The NMC includes the ADC, the hardware implementation of the ANN (neurromorphic chip), the device for encryption and data transmission, the device for adaptive control of the machining process and the CPU for coordinating and controlling all the elements (Fig. 7). The ADC input receives data from a distributed sensor system where digitization takes place. The received data arrive at the entrance of the neurochip. There is their classification and definition of the state of the technological system. Based on the obtained data, real-time processing modes are adjusted using an adaptive device. All received data is sent to the database in an encrypted form. The use of industrial neurochips allows for high system stability, performance and low power consumption. 


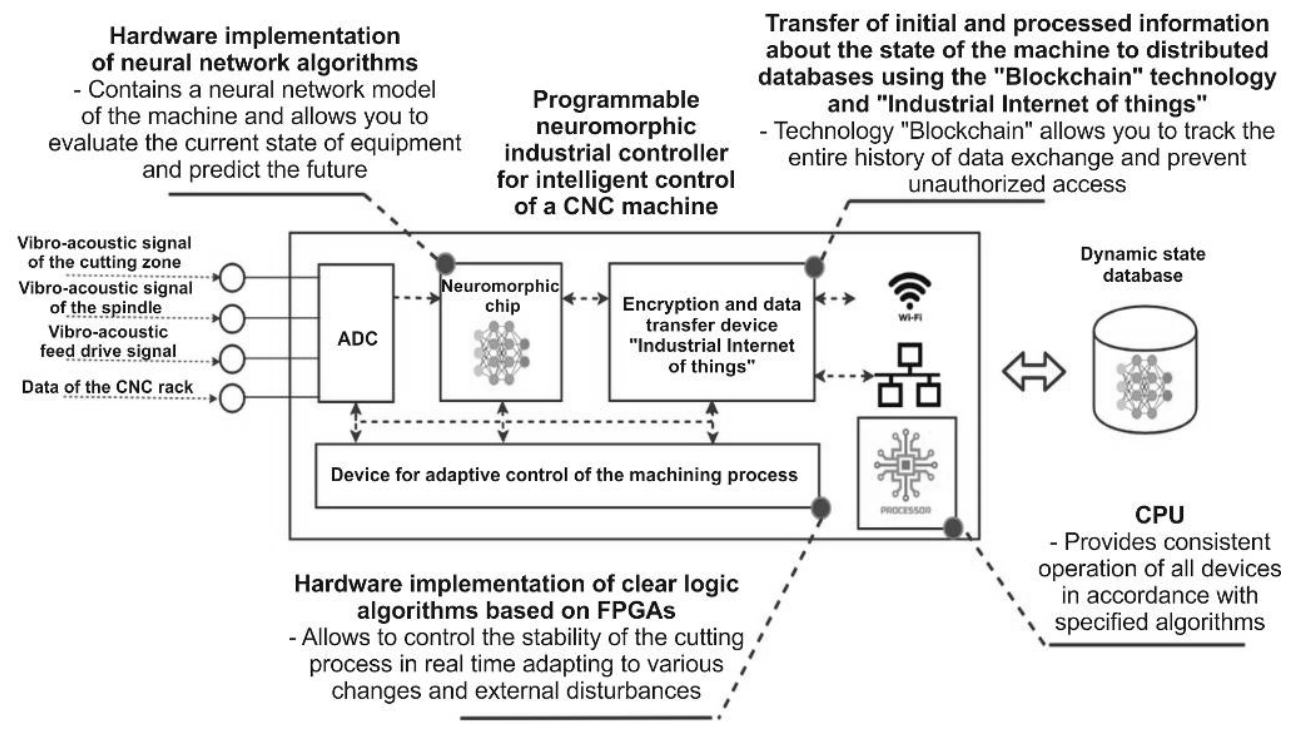

Fig. 7. Programmable neuromorphic controller for industrial design of the NSTU. R.E. Alekseeva

The controller consists of two principal modules. The first module makes it possible to analyze the dynamic stability of the cutting process itself, as an auto oscillatory system, applying the approaches of nonlinear dynamics and artificial intelligence. The second module allows analyzing the technical condition of such units as: gearboxes, bearings, electric motors, ball screws, guides, etc. Here, mostly deterministic universal dependencies and algorithms are used. AI approaches are used as a complement and take into account the peculiarities of each equipment.

\section{Advantages of digitizing machine-building productions}

- Reduction of preparation time to $30 \%$ due to optimization of processing modes and correction of control programs based on neural network modeling of machining processes taking into account the current state of the machine;

- Increase tool life up to 4 times due to optimized modes and processing conditions;

- Product quality management at the stage of technological preparation of production;

- Significant reduction of equipment downtime due to constant monitoring and forecasting of its technical condition;

- Constant collection and accumulation of knowledge;

- Sharing knowledge between the equipment allows to speed up the process of learning neural network models;

- Identify the reasons for downtime;

- Invariance to the equipment manufacturer

\section{Conclusion}

The transition to the digital industry is accompanied, first of all, by the modernization of existing CNC machines to cyber-physical systems that are characterized by high adaptive and intelligent capabilities. The main approaches at the same time are: artificial neural networks, technologies for storing and processing big data, industrial Internet of things and encryption technologies - «Block chain». It is also necessary to develop new industrial 
processors with a neuromorphic structure, allowing to manage the machines in the group of technological systems with the possibility of knowledge sharing.

\section{References}

1. Yu.G. Kabaldin, D.A. Shatagin, M.S. Anosov, P.V. Kolchin, A.M. Kuzmishina, Artificial intelligence and cyber-physical machining systems in digital production: monograph (Nizhny Novgorod state. tech. un-t them. R.E. Alekseeva, Nizhny Novgorod, 2018)

2. Yu.G. Kabaldin, S.V. Bilenko, S.V. Seryi, Control of dynamic processes in technological systems of machining based on artificial intelligence (Komsomolsk-onAmur state. tech. un-t, Komsomolsk-on-Amur, 2003)

3. Yu.G. Kabaldin, I.L. Laptev, D.A. Shatagin, S.V. Seryi, Bulletin of Machine Building, 8, 80 (2014)

4. Yu.G. Kabaldin, I.L. Laptev, D.A. Shatagin, V.O. Zotov, S.V. Seryi, Machine building: a network electronic scientific journal, 2, 47 (2014)

5. Yu.G. Kabaldin, I.L. Laptev, D.A. Shatagin, Yu.V. Vytnov, S.V. Golubev, Proceedings of the NSTU, 5, 114 (2013)

6. Yu.G. Kabaldin, D.A. Shatagin, I.L. Laptev, D.A. Sidorenkov, Izvestiya Vysshikh Uchebnykh Zavedenii, 10, 49 (2016) 